\title{
235 NOVEL BIPARATOPIC TIM-3 ANTIBODY EFFECTIVELY BLOCKS MULTIPLE INHERENT LIGANDS AND ACTIVATES ANTI-TUMOR IMMUNITY
}

Yuji Mishima*, Kanto Nakajima, Mamoru Shiraishi, Haruka Matsumura, Takahiko Aramaki, Noriko Matsumoto, Norihiro Nakamura. Brightpath Biotherapeutics, Kawasaki, Japan

Background T cell immunoglobulin and mucin domain-containing protein 3 (TIM-3) is a part of modules expressed on dysfunctional or exhausted $\mathrm{T}$ cells as well as dendritic cells and has emerged as a target for several therapeutic antibodies that are under clinical development. Co-blockade of TIM-3 and PD-1 results in tumor regression in preclinical models and improves anticancer $\mathrm{T}$ cell responses in patients with advanced cancers. TIM-3 has been reported to have multiple ligands including galectin-9, phosphatidylserine, CEACAM-1 and HMGB1, which bind to different regions on the extracellular domain of TIM-3. Most of the TIM-3 antibodies developed to date are intended to inhibit phosphatidylserine that binds to the pocket in TIM-3 immunoglobulin V domain. Galectin-9 binds to carbohydrate motifs on the opposite side of phosphatidylserine-binding site in immunoglobulin $\mathrm{V}$ domain and thereby induces cell death in TIM-3 $+\mathrm{T}$ cells. We report herein novel antibodies that block TIM-3 binding to multiple ligands including these two important ligands simultaneously.

Methods Anti-TIM-3 antibodies were generated by immunizing mice with a purified recombinant TIM-3 protein and TIM-3expressing mammalian cell line. Phage display libraries were constructed using cDNAs of splenocytes and lymph node cells of the immunized mice, then subjected to the biopanning using recombinant TIM-3 proteins. After analyzing specificities and affinities to the TIM-3 protein, scFvs obtained were classified by epitope bin and inhibitory effects on TIM-3 binding to the multiple ligands. The scFvs were converted to scFv-Fc to generate biparatopic (bispecific) antibodies.

Results At least five classes of TIM-3 antibodies were obtained, and each class was grouped into different epitope bins and has unique inhibitory profiles for multiple ligands of TIM-3. Their biparatopic (bispecific) forms were produced from the $\mathrm{scFv}$ clones and subjected to the analyses of TIM-3 binding, inhibition of ligand binding, and immune activation. As expected, the biparatopic antibodies that recognize two different epitopes showed higher affinity and specificity to TIM3 than monospecific forms. A lead biparatopic antibody that block the binding of TIM-3 to galectin-9 and phosphatidylserine showed remarkable potency on $\mathrm{T}$ cell activation, protection from exhaustion and apoptotic cell death of $\mathrm{T}$ cells as well as more potent anti-tumor efficacy.

Conclusions This study demonstrates the successful development of a novel biparatopic antibody that blocks the binding of TIM-3 to phosphatidylserine and galectin-9 simultaneously. The antibody shows the advantages over conventional TIM-3 antibodies in reducing $\mathrm{T}$ cell exhaustion and potentially manipulated for the development of human monoclonal antibodies for therapeutic treatment of cancer.

http://dx.doi.org/10.1136/jitc-2021-SITC2021.235 